\title{
A seismic probability map
}

\author{
J. M. MuvuerA
}

Ricevuto il 3 Ottobre 1964

\begin{abstract}
Summary. - The material included in former two papers (ST) and EF) which summs 3307 shocks corresponding to 2360 years, up to 1960 , was reduced to a 50 year's period by means the weight obtained for each epoch. The weithing factor is the ratio 50 and the amount of years for every epoch.

The frequency has been referred over basis VII of the international seismic scale of intensity, for all cases in which the earthquakes are equal or greater than VI and up to IX. The sum of products: frequency and parameters previously exposed, is the probable frequency expected for the 50 years period.

On each active small square, we have made the corresponding computation and so we have drawn the Map No 1 , in percentage. The epicenters with intensity since $\mathrm{X}$ to $\mathrm{XI}$ are plotted in the Map No 2, in order to present a complementary information.

A table shows the return periods obtained for all data (VII to XI), and after checking them with other computed from the first up to last shock, a list includes the probable approxinate return periods estimated for the area.

The solution, we suggest, is an appropriated form to express the seismic contingent phenomenon and it inproves the conventional maps showing the equal intensity curves corresponding to the maximal values of given side.
\end{abstract}

Riassunto. - Il materiale presentato nelle due ultime note (SD e EF), che comprende 3307 scosse corrispondenti a 2360 anni, fino al 1960 , es stato ridotto ad un cinquantennio tenendo conto del fattore peso ottenuto per ogni epoca.

Ia frequenza e stata riferita al VIIo di intensita della scala internazionale, per tutti i casi in cui detta intensita era compresa fra il VIo ed il IXo. Ia somma dei prodotti della frequenza per i parametri sucitati, e la frequenza probabile che ci si aspetta per un periodo di cinquanta anni.

Su ogni quadretto abbiamo fatto il calcolo corrispondente, disegnando cosi la cartina $n^{\circ} 1$, in percentuali. Nella cartina $n^{\circ} 2$ sono stati disposti gli epicentri dei terremoti con intensita dal $\mathrm{X}^{\circ}$ all' $\mathrm{XI}^{\circ}$, allo scopo di fornire ulteriori informazioni. 
Una tabella riporta $\mathrm{i}$ "periodi di ritornon ottenuti per tutti i dati (dal VIIo al $\mathrm{IX}^{\circ}$ ), dopo averli controllati con quelli calcolati dalla prina all'ultima scossa; la tabella $V$ comprende i "periodi di ritorno" probabili e approssimati, valutati per la regione presa in esame.

Riteniamo questa soluzione piu appropriata per esprimere gli eventuali fenomeni sismici, in modo da migliorare le carte convenzionali rappresentanti le isosiste corrispondenti ai massimi valori dell'intensita dei terremoti.

ExTracro. - EI material presentado en dos artículos anteriores (SD) y EF), que suma 3307 sacndidas correspondientes a 2360 años, hasta 1960 , hil sido reducido a un periodo de 50 años mediante el peso obtenido para carla epoca. El factor de peso es la relacion entre 50 y el numero de anos del intervalo de cacla epoea.

Ia frecuencia ha sido referida a base VII de la escalia internacional de grados de intensidad sísmica, para todos los casos de terremotos con intensidad igual o mayor que grado VI, y hasta el IX.

Ja suma de los productos de frecnencia por los parametros anteriormente mencionalos es la frecuencia probable que se espera para el periodo de 50 años.

Para cada cuadricula activa se ha hecho el ealculo correspondiente $y$, con esos resultatos, se ha trazado el Mapa l, en tanto por ciento. Los epicentros de intensidad desile X a XI se han dibujado en el ILapa 2, con objeto de presentar una informacion complementaria.

En una tabla, se consignan los periodos de retorno para todos los datos (VII a XI) y una lista incluye los periodos de retorno, aproximados y probables, que se estiman para la region, despues de haber comparado los resultados de la tabla con los calculados directamente entre el mis remoto y el ultimo de los terremotos de la misma intensidad.

Se sugiere que la solucion es apropiada para expresar el fenomeno aleatorio sísmico, y que mejora los mapas eonvencionales de isosistas trazados con los valores maximos de intensidad.

\section{Purpose.}

In former two palpers (6, SD; 7 , EF) we have presented several characters about the seismicity of the area $35^{\circ}-44^{\circ} \mathrm{N}$ and $10^{\circ} \mathrm{W}-5^{\circ} \mathrm{E}, \mathrm{Gr}$. This material is applied now to draw a map which improves the eonventional ones showing the equal-intensity curves (iso-lines) corresponding to the maximal values of given side, because probability is, surely, a mole accurate form to express the seismic contingent phenomenon.

The map is appropriate for earthqu ske engineering and for comparing with tectonic or gravimetric informations. 


\section{Material USED.}

Table I resumes the data and its weight, which is as the ratio of 50 (period taken for establishing the probable frequency computation) and the number of years for each epoch. We have selected 3307 shocks, corresponding to 2360 years, and the datar were reduced to at 50 years period, as it was explaines in EF.

Table I - Summarx.

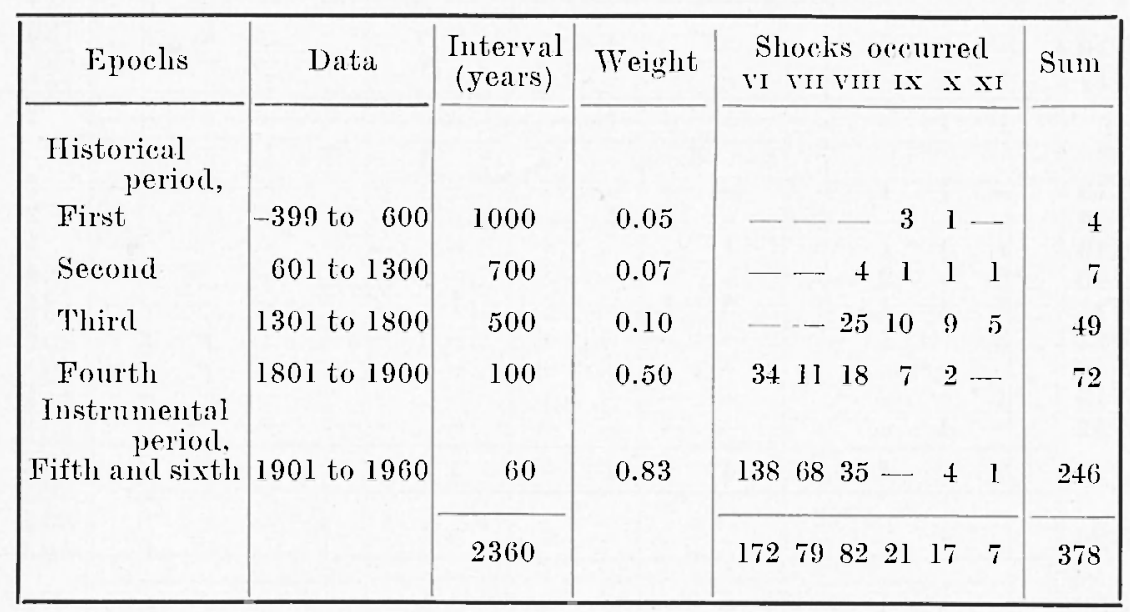

Table II includes the shocks located in the area, with an intensity larger than $\mathrm{VI}^{\circ}$ of the international seismic intensity scale.

\section{Method used.}

A parabola and a simplified linear expression for the whole investigated area were obtained in $\mathrm{EF}$, for adjusting the regression of $\log \mathrm{N}$ and $\mathrm{m}$. The last simple expression will be used for each small square on the area, after adding $\log 50$, because 50 is the number of years taken as the period for defining the probable frequency.

The correspondence between $\mathrm{I}_{0}$ and $\mathrm{n}$, was obtained in SD from the CSE formula, assuming a normal depth. Frequency is referred to basis VII degree for shocks of intensity VI to IX; while the epicenters corresponding to $\mathrm{X}^{\circ}$ and $\mathrm{XI} \mathrm{I}^{\circ}$ are drawn on an independent map. The influence on the numerical values of those last shocks should increase 
Table II - Shocks, vi- IX, AND EXPECtant for 50 years By $10^{3} \mathrm{~km}^{2}$.

\begin{tabular}{|c|c|c|c|c|c|c|c|c|c|c|c|c|c|c|c|c|}
\hline \multirow{2}{*}{\multicolumn{2}{|c|}{ Region map }} & \multicolumn{4}{|c|}{ 6th, 5th } & \multicolumn{4}{|c|}{4 th } & \multicolumn{2}{|c|}{ 3rd } & \multicolumn{2}{|c|}{ 2nd } & \multirow{2}{*}{$\begin{array}{l}\text { 1st } \\
\text { IX }\end{array}$} & \multirow{2}{*}{ Sum } & \multirow{2}{*}{ W.E. } \\
\hline & & VI & VII & VIII & IX & VI & VII & VIII & IX & VIII & IX & VII & IX & & & \\
\hline \multirow[t]{4}{*}{1} & T01 & 1 & - & - & - & - & - & - & - & - & - & - & - & - & 1 & 0.1 \\
\hline & W04 & - & 1 & - & - & 1 & - & - & - & - & - & - & - & - & 2 & 0.4 \\
\hline & X05 & - & 1 & - & - & - & - & - & - & - & - & - & - & - & 1 & 0.3 \\
\hline & & 1 & 2 & - & - & 1 & - & - & - & - & - & - & - & - & 4 & \\
\hline \multirow{5}{*}{2} & W08 & 1 & - & - & - & - & - & - & - & - & - & - & - & - & 1 & 0.1 \\
\hline & W10 & - & - & - & - & - & - & - & - & - & - & - & 1 & - & 1 & 0.2 \\
\hline & W13 & - & 1 & - & - & - & - & - & - & - & - & - & - & - & 1 & 0.3 \\
\hline & W14 & 1 & - & - & - & - & - & - & - & - & - & - & - & - & 1 & 0.1 \\
\hline & & 2 & 1 & - & - & - & - & - & - & - & - & - & 1 & - & 4 & \\
\hline \multirow[t]{3}{*}{3} & T16 & - & 1 & - & - & - & - & 1 & - & - & - & - & - & - & 2 & 0.9 \\
\hline & W17 & 1 & - & - & - & - & - & - & - & - & - & - & - & - & 1 & 0.1 \\
\hline & & 1 & 1 & - & - & - & - & 1 & - & - & - & - & - & - & 3 & \\
\hline \multirow[t]{10}{*}{4} & T19 & - & 1 & 1 & - & - & - & - & - & - & - & - & - & - & 2 & 1.3 \\
\hline & T22 & - & - & - & - & - & 1 & 1 & - & - & - & - & - & - & 2 & 0.8 \\
\hline & U19 & 1 & 1 & 1 & - & 1 & - & - & - & - & - & - & - & - & 4 & 1.5 \\
\hline & U20 & 2 & - & 2 & - & - & - & - & - & - & - & - & - & - & 4 & 2.3 \\
\hline & U21 & l & 4 & 1 & - & 3 & 1 & - & - & 1 & - & - & - & - & 11 & 3.0 \\
\hline & U22 & 2 & - & 1 & - & - & - & - & - & - & - & - & - & - & 3 & 1.3 \\
\hline & W18 & - & - & - & - & - & - & - & - & - & - & - & - & - & - & - \\
\hline & W19 & 1 & - & - & - & - & - & - & - & - & - & - & - & - & 1 & 0.1 \\
\hline & W 22 & - & 1 & - & - & - & - & - & - & - & - & - & - & - & 1 & 0.3 \\
\hline & & 7 & 7 & 6 & - & 4 & 2 & 1 & - & 1 & - & - & - & - & 28 & \\
\hline 5 & W26 & - & 1 & - & - & - & - & - & - & - & - & - & - & - & 1 & 0.3 \\
\hline \multirow[t]{3}{*}{6} & U30 & - & 1 & 1 & - & - & - & - & - & - & - & - & - & - & 2 & 1.3 \\
\hline & $\mathrm{X} 30$ & - & 1 & - & - & - & - & - & - & - & - & - & - & - & 1 & 0.3 \\
\hline & & - & 2 & 1 & - & - & - & - & - & - & - & - & - & $\overline{-}$ & 3 & \\
\hline \multirow[t]{9}{*}{7} & MO1 & - & - & - & - & 1 & - & - & - & - & - & - & - & - & 1 & 0.1 \\
\hline & N03 & 1 & - & - & - & - & - & - & - & - & - & - & - & - & 1 & 0.1 \\
\hline & N06 & - & - & - & - & - & - & 1 & - & - & - & - & - & - & 1 & 0.6 \\
\hline & R0I & - & 1 & - & - & - & - & - & - & - & - & - & - & - & 1 & 0.3 \\
\hline & $\mathrm{R} 03$ & 3 & - & - & - & - & - & - & - & 1 & - & - & - & - & 4 & 0.4 \\
\hline & R04 & 1 & - & - & - & - & - & - & - & - & - & - & - & - & 1 & 0.1 \\
\hline & R06 & 1 & - & - & - & - & - & - & - & - & - & - & - & - & 1 & 0.1 \\
\hline & S03 & 3 & - & - & - & - & - & - & - & - & - & - & - & 1 & 4 & 0.6 \\
\hline & & 9 & 1 & - & - & 1 & - & 1 & - & 1 & - & - & - & 1 & $\overline{14}$ & \\
\hline \multirow[t]{5}{*}{8} & K 17 & 1 & - & - & - & - & - & - & - & - & - & - & - & - & 1 & 0.1 \\
\hline & N15 & 1 & - & - & - & - & - & - & - & & - & - & - & - & 1 & 0.1 \\
\hline & S13 & - & - & - & - & 1 & - & - & - & - & - & - & - & - & 1 & 0.1 \\
\hline & U11 & - & - & - & - & - & - & - & - & - & - & - & - & - & - & - \\
\hline & & 2 & - & - & - & 1 & - & - & - & - & - & - & - & - & 3 & \\
\hline 9 & N18 & - & - & - & - & 1 & - & - & - & - & - & - & - & - & 1 & 0.1 \\
\hline & $\mathrm{N} 21$ & 1 & - & - & - & - & & & - & & - & - & - & - & & \\
\hline & $\mathrm{P} 22$ & - & - & - & - & 1 & - & - & $=$ & - & $\overline{-}$ & - & - & - & 1 & $\begin{array}{l}0.1 \\
0.1\end{array}$ \\
\hline & R18 & 1 & 1 & - & - & - & - & - & - & - & - & - & - & - & 2 & \\
\hline & S17 & - & 1 & - & - & - & - & - & - & - & - & - & - & - & 1 & 0.3 \\
\hline & $\mathrm{S} 19$ & 1 & - & - & - & - & - & - & - & - & - & - & - & $1-$ & 1 & 0.1 \\
\hline & & 3 & 2 & - & - & 2 & - & - & - & - & - & - & - & - & 7 & \\
\hline
\end{tabular}


Table II: continuation.

\begin{tabular}{|c|c|c|c|c|c|c|c|c|c|c|c|c|c|c|c|c|}
\hline \multirow{2}{*}{\multicolumn{2}{|c|}{ Region map }} & \multirow{2}{*}{\multicolumn{4}{|c|}{$\begin{array}{l}6 \text { th, } 5 \text { th } \\
\text { VI VII VIII Ix }\end{array}$}} & \multicolumn{4}{|c|}{ 4th } & \multicolumn{2}{|c|}{$3 \mathrm{rd}$} & \multicolumn{2}{|c|}{ 2nd } & \multirow{2}{*}{$\begin{array}{l}\text { lst } \\
\text { IX }\end{array}$} & \multirow{2}{*}{ Sum } & \multirow{2}{*}{ W. E } \\
\hline & & & & & & VI & VII & VIII & $\mathrm{IX}$ & VIII & $\mathrm{IX}$ & VIII & $\mathrm{IX}$ & & & \\
\hline \multirow[t]{14}{*}{10} & $\mathrm{R} 24$ & I & - & - & - & - & - & - & - & - & - & - & - & & I & 0.1 \\
\hline & $\mathrm{R} 25$ & 4 & - & - & - & 1 & - & - & - & - & - & - & - & - & 5 & 0.6 \\
\hline & $\mathrm{S} 23$ & - & - & - & - & 1 & - & - & - & - & - & - & - & - & 1 & 0.1 \\
\hline & S24 & 2 & - & - & - & - & - & - & - & - & - & - & - & - & 2 & 0.3 \\
\hline & $\mathrm{S} 25$ & - & 1 & - & - & -. & - & 1 & - & I & - & - & - & - & 3 & 1.1 \\
\hline & S26 & 1 & - & - & - & - & - & - & - & I & - & - & - & - & 2 & 0.3 \\
\hline & '1'23 & 1 & - & - & - & - & - & - & - & 1 & - & - & - & - & 2 & 0.3 \\
\hline & T24 & 1 & - & - & - & - & I & - & - & - & - & - & - & - & 2 & 0.3 \\
\hline & T25 & - & - & - & - & I & - & - & - & -- & - & - & - & - & 1 & 0.1 \\
\hline & T26 & - & - & - & - & - & - & - & - & - & 3 & - & - & - & 3 & 0.7 \\
\hline & T27 & - & $\mathrm{I}$ & - & - & - & - & - & - & - & - & - & - & - & 1 & 0.3 \\
\hline & U23 & - & - & - & - & I & 1 & - & - & - & - & - & - & - & $\underline{?}$ & 0.3 \\
\hline & U24 & - & - & 1 & - & - & - & - & - & - & - & - & - & - & 1 & 1.0 \\
\hline & & 10 & 2 & 1 & - & 4 & 2 & I & - & 3 & 3 & - & - & - & 26 & \\
\hline \multirow[t]{11}{*}{ II } & J02 & - & - & 1 & - & - & - & - & - & - & - & - & - & - & I & 1.0 \\
\hline & J04 & 1 & - & - & - & - & - & - & - & - & - & - & - & - & I & 0.1 \\
\hline & J05 & I & 1 & - & - & 1 & - & - & - & - & - & - & - & - & 3 & 0.5 \\
\hline & K02 & 3 & 1 & 1 & - & I & - & 1 & - & 1 & 1 & - & - & - & 9 & 2.8 \\
\hline & K03 & 2 & - & - & - & - & - & - & - & - & - & - & - & - & 2 & 0.3 \\
\hline & $\mathrm{K} 04$ & 1 & - & - & - & - & - & - & - & - & - & - & - & - & 1 & 0.1 \\
\hline & K05 & - & I & - & - & - & - & - & - & - & - & - & - & - & 1 & 0.3 \\
\hline & L 02 & 2 & - & - & - & - & - & - & - & - & - & - & - & - & 2 & 0.3 \\
\hline & $\mathrm{I}, 03$ & 1 & I & - & - & - & - & - & - & - & - & - & - & - & 2 & 0.5 \\
\hline & I.05 & 1 & - & - & - & - & - & - & - & - & - & - & - & - & 1 & 0.1 \\
\hline & & $\mathrm{I} 2$ & 4 & 2 & - & 2 & - & 1 & -- & 1 & $I$ & - & - & - & 23 & \\
\hline 12 & K09 & - & - & 1 & - & - & - & - & - & - & - & - & - & - & I & 1.0 \\
\hline \multirow[t]{7}{*}{13} & D04 & 1 & - & - & - & - & - & - & - & - & - & - & - & - & I & 0.1 \\
\hline & E03 & $i$ & - & - & - & - & - & - & - & - & - & - & - & - & I & 0.1 \\
\hline & $\mathrm{E} 04$ & 2 & - & - & -. & I & - & - & - & 2 & - & - & - & - & 5 & 0.7 \\
\hline & $\mathrm{E} 05$ & - & - & - & - & - & - & - & - & - & - & - & - & - & - & - \\
\hline & II03 & - & - & - & - & - & - & - & $\overline{-}$ & - & $\overline{-}$ & - & - & - & $\overline{1}$ & $\overline{0}$ \\
\hline & $\begin{array}{l}\text { H04 } \\
\text { H05 }\end{array}$ & $\begin{array}{l}\text { l } \\
\text { I }\end{array}$ & - & $\overline{-}$ & $\begin{array}{l}- \\
-\end{array}$ & 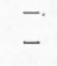 & $\overline{-}$ & $\overline{-}$ & $\overline{-}$ & - & $\overline{-}$ & $\overline{-}$ & $\begin{array}{l}- \\
-\end{array}$ & $\overline{-}$ & $\begin{array}{l}1 \\
1\end{array}$ & 0.1 \\
\hline & & 6 & - & - & - & I & - & - & - & 2 & - & - & - & - & 9 & \\
\hline \multirow[t]{5}{*}{14} & D06 & - & I & -. & - & - & - & - & - & 2 & - & - & - & - & 3 & 0.6 \\
\hline & D08 & I & - & - & - & - & - & - & - & - & - & - & - & - & I & 0.1 \\
\hline & E06 & I & - & - & - & - & - & - & - & - & - & - & - & - & I & 0.1 \\
\hline & E 09 & - & - & - & - & - & - & - & - & - & - & I & - & 1 & I & 0.1 \\
\hline & & 2 & 1 & - & - & - & - & - & - & 2 & - & 1 & - & 1 & 6 & \\
\hline
\end{tabular}


Table II: continuation.

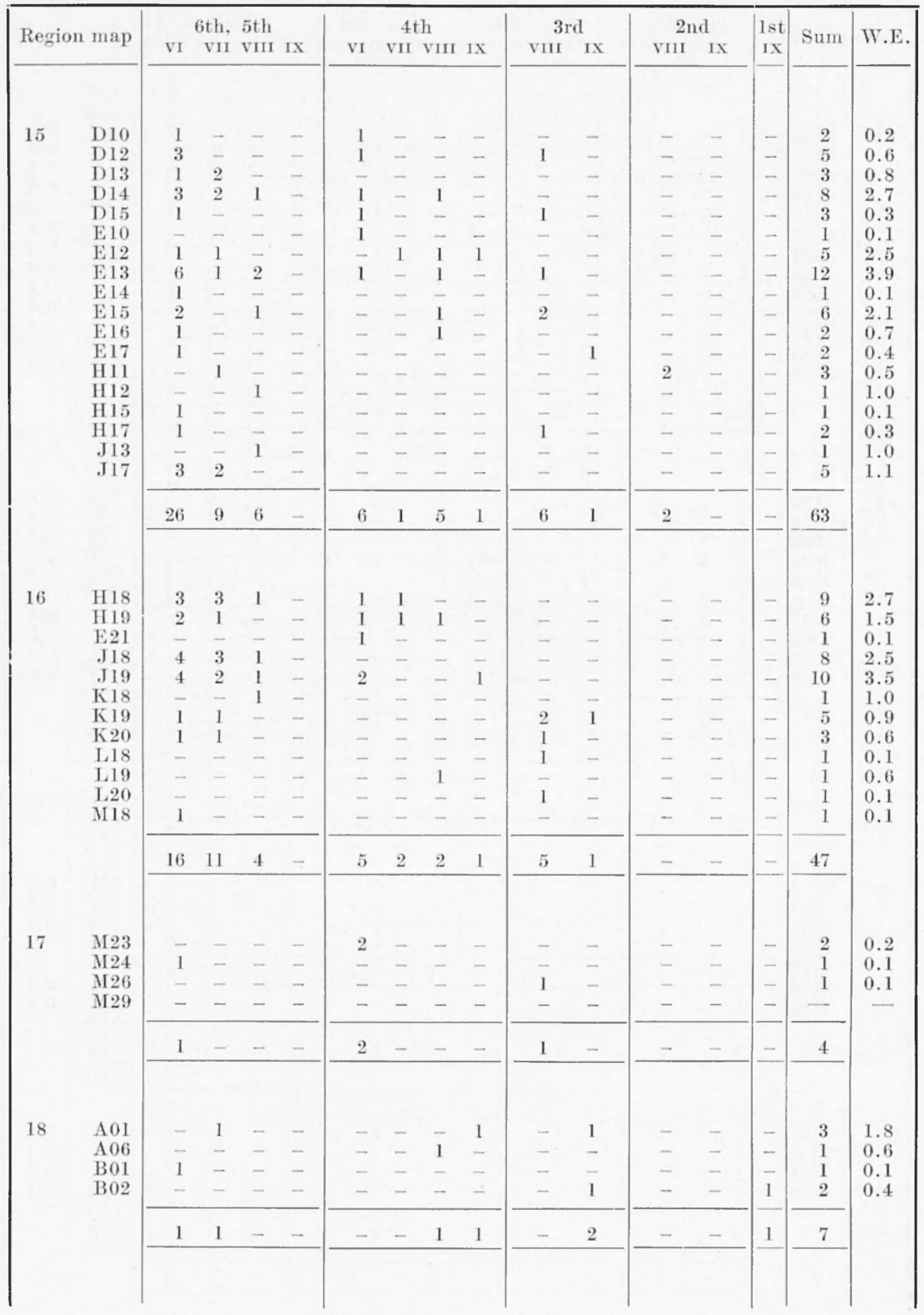


Table II: continuation.

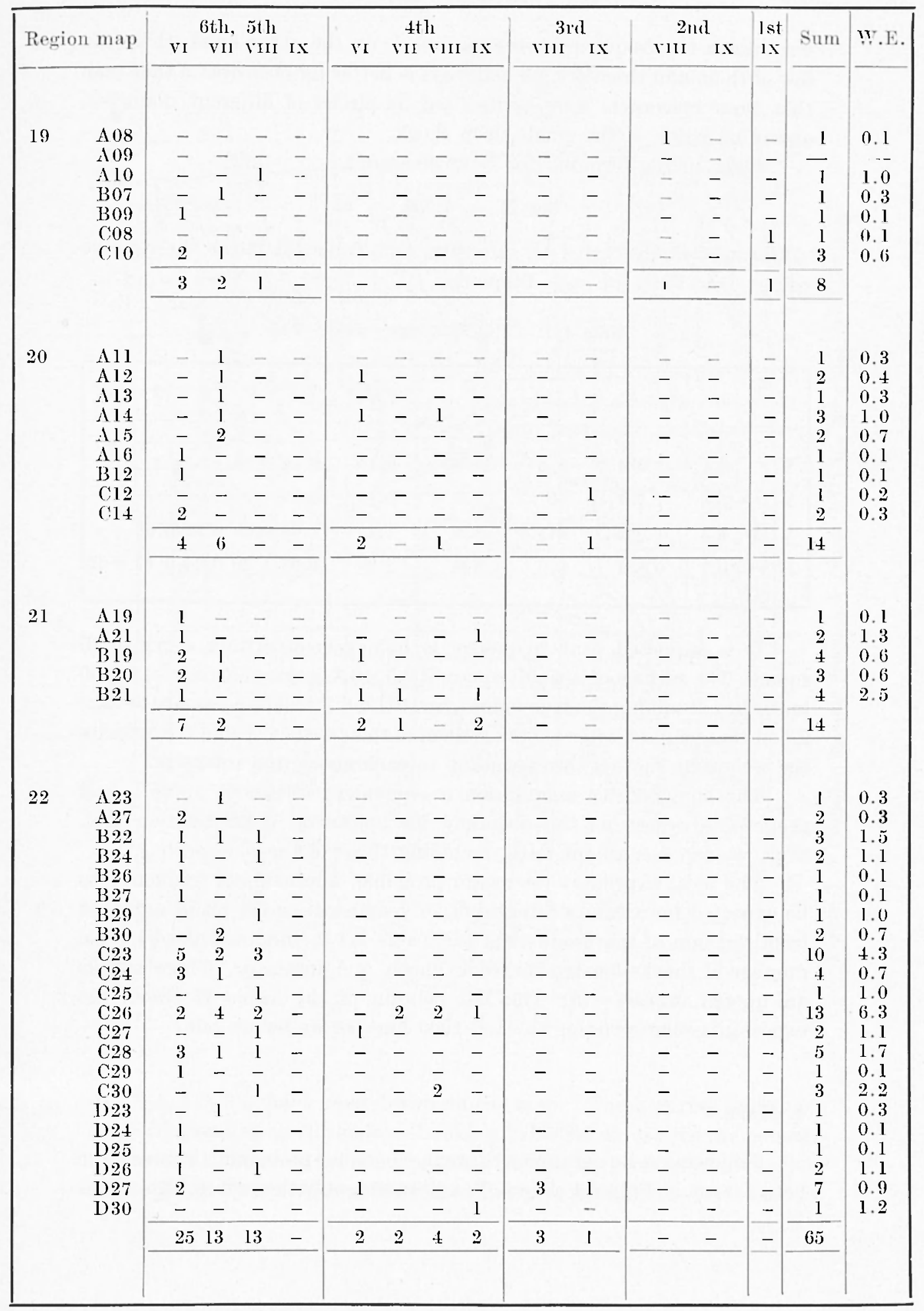


very much the frequency percentage and, on the other hand, there are few of them and therefore, we believe it is better for clearness of that map that these epicenters were symbolized by circles of different diameter, exception made of the single deep shock.

Thus, in the formula (for $\mathrm{N}$ in 50 years),

$$
\log \mathrm{N}=7.551-\mathrm{m}
$$

magnitude is substituted by intensity, and Table III shows the relative values over VII', for each frequency.

Table III - Paraneters, BasIS VIIo.

\begin{tabular}{|c|c|c|c|c|c|c|c|c|c|c|}
\hline $\mathrm{I}_{0}$ & $m$ & $\log N_{50}$ & $1.751-$ & $\mathrm{C}$ & $10^{3} \mathrm{k}^{2} \mathrm{n}^{2}$ & 5,6 & 4 & 3 & 2 & 1 \\
\hline VIo & 5.4 & 2.151 & 1.6 & 0.4 & 0.16 & 0.13 & 0.08 & - & - & - \\
\hline VIIo & 5.8 & 1.751 & 0 & 1.0 & 0.40 & 0.33 & 0.20 & -- & - & 一 \\
\hline VIIIo & 6.3 & 1.251 & 0.5 & 3.0 & 1.20 & 1.00 & 0.60 & 0.12 & 0.08 & - \\
\hline I Xo & 6.7 & 0.851 & 0.9 & 6.0 & 2.40 & 1.99 & 1.20 & 0.24 & 0.17 & 0.12 \\
\hline
\end{tabular}

It is supposed that frequency is homogeneous within every small square. The surface of a small square is $0.5 \times 0.5$ geographic degrees $(2,500$ $\mathrm{km}^{2}$ ). For obtaining the true values, by $10^{3} \mathrm{~km}^{2}$, it will be nccessary to take $2 / 5$ of these values, after affecting them of their weight. Table III includes the weighting factors corresponding to each epoch and intensity.

The homogeneity assumption is equivalent to accept up to a half geographic degree as the accuracy for epicentral determination and, thus, we can use all the data, including those of the old epochs.

The local expectant (or mean, probable, approximate frequency to be expected for a future interval of 50 years) will be the result obtained from the sum of the products of the Table III factors multiplied by the number of shocks located for each epoch and intensity. These shocks are quoted in Table II. The last column of the Table II shows the expectants corresponding to each $1000 \mathrm{~km}^{2}$, as we before said.

\section{Maps.}

Following a topographic criterion, the equal-probability-curves have been drawn, as Figure 1 shows. For clearness, only the curves with values 


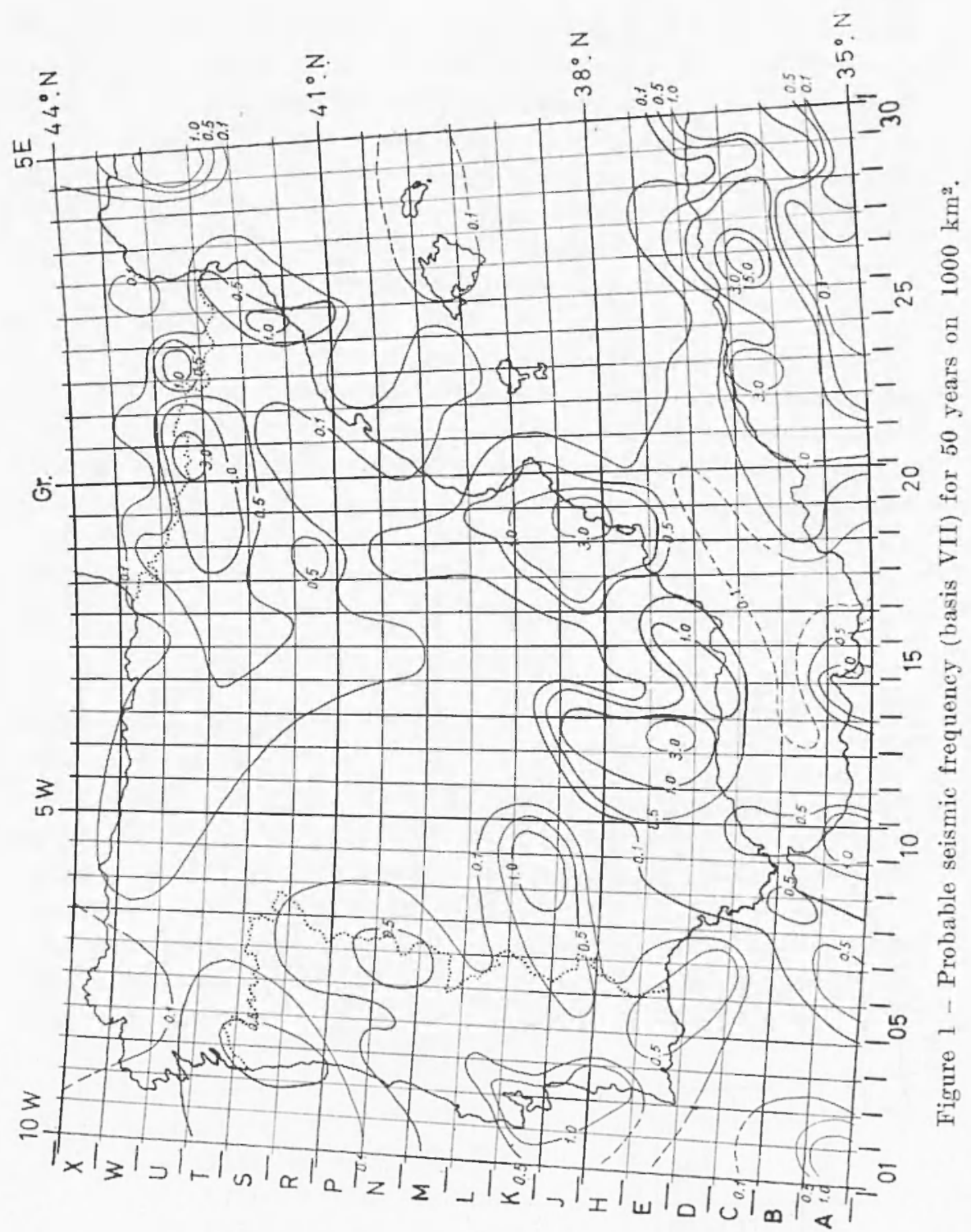

$0.1,0.5,1.0,3.0$, have been drawn on the map. These curves surround the corresponding small squares. Looking Table III we can see the pereentage over VII ${ }^{\circ}$ referred to $1000 \mathrm{~km}^{2}$ and with weight, versus a whole degree of one shock by a whole small square. In consequence, it is very easy to 


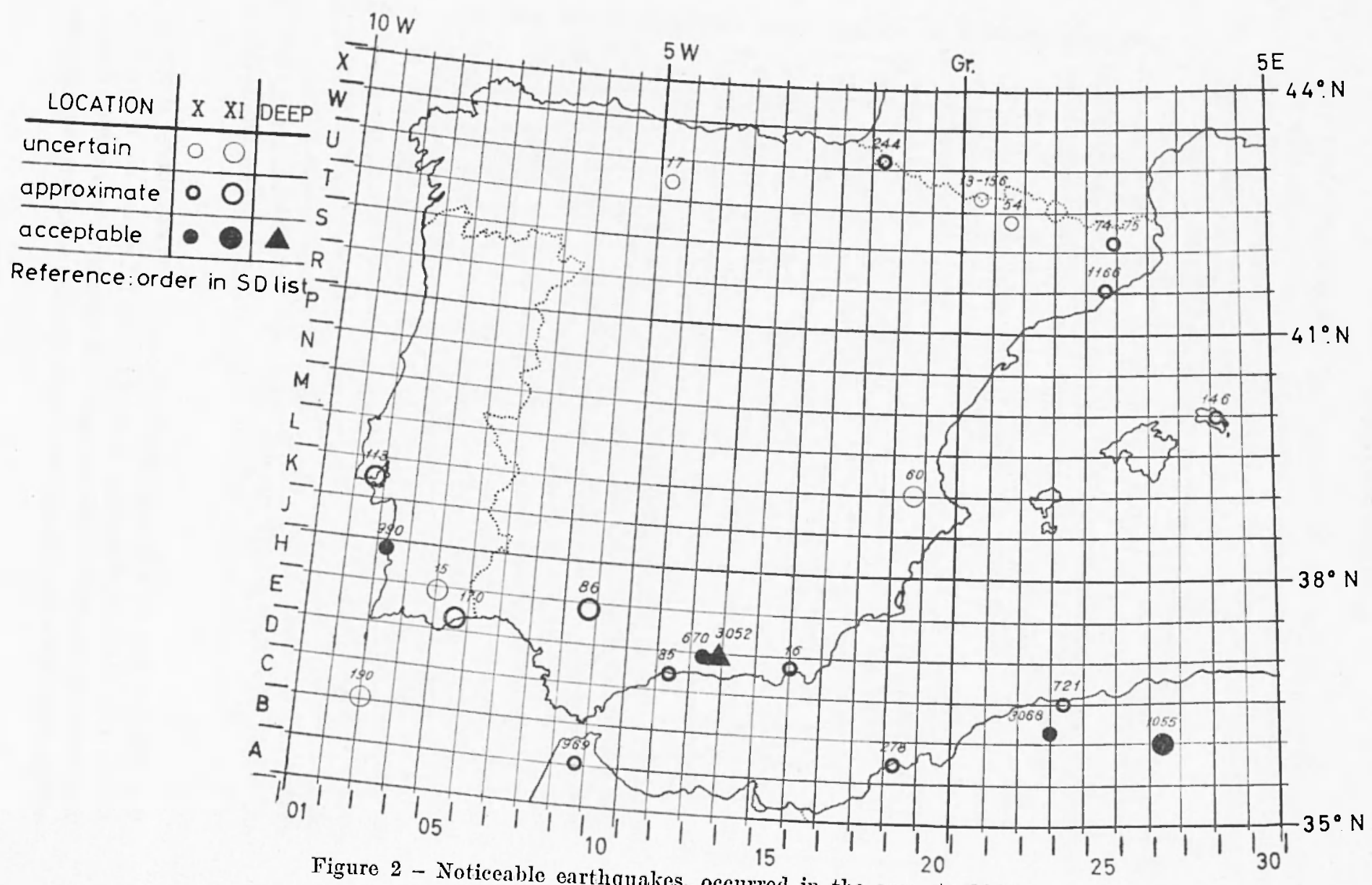

Figure 2 - Noticeable earthquakes, oceurred in the area, to 1960 
draw a map for equal-probable-intensity curves (probability iso-lines). The eurves could be drawn jointing the centers of ach active small squares, which have the same value of intensity.

Figure 2 presents the $\mathrm{X}^{\circ}$ and XIo epicenters and also the decp shock of Andalucia. These are the noticeable shocks oceurred in the area till 1960 and they are listed in the Table IV. We use the intensity scale suggested by Richter, which takes in arcount four references for diflerent kinds of masonry; other similar seale was compilated by MLedveder, Sponheuer and Fianik, in 1962, and it was proposed by European Seismological Commission, CSE.

Certainly, it is preferable to have a map of iso-probability-lines for seismic zone; Figure 1 is also useful for this purpose. When the expectant is smaller than 0.16 (intensity minor than VIo for a given small square) seismicity is of course not appreciable. For expertant from 0.16 to 2.4 (VIo-IXo), seismic activity is progressively increasing; but, if the expectant is larger than 2.4 (intensity higher than $\mathrm{IX}^{\circ}$, for a certain small square) it is absolutely necessary to take into account seismic activity, which may be dangerous for people and buildings.

\section{RETURN PERIODS.}

As an additional information which could help for further studies and applications, Table $Y$ has witten the return periods for the selected quakes from VII up to XI dg, occurred for every 22 regions established in the area (see EF) and also for the whole area.

The return period (inverted of the probability or interval expressed in years during which a quake of a given degree is to be expected) is deduced from the data grouped in the following form:

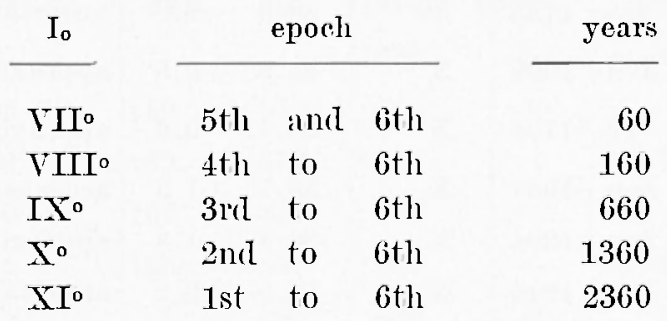

These intervals, of the last columm, divided by the number of earthqualkes corresponding to each active small square (Table II) give the values 
Table IV - Noticeable earthquakes in the area ( $\mathrm{x}^{0}, \mathrm{xi}^{\circ}$ and deep one).

\begin{tabular}{|c|c|c|c|c|c|c|c|c|c|c|c|}
\hline \multicolumn{2}{|c|}{ Regrion map } & \multicolumn{3}{|c|}{ Epocli } & \multirow{2}{*}{$\begin{array}{c}\text { Data } \\
\\
949\end{array}$} & \multirow{2}{*}{$\begin{array}{l}\mathrm{I}_{0} \\
\mathrm{X}\end{array}$} & \multicolumn{2}{|c|}{ Coordinates } & \multirow{2}{*}{$\begin{array}{l}\text { Location } \\
\text { uncertain }\end{array}$} & \multirow{2}{*}{$\begin{array}{r}\begin{array}{c}\text { Order } \\
\text { SD }\end{array} \\
17\end{array}$} & \multirow{2}{*}{ Remarks } \\
\hline 8 & U11 & 2 & & & & & 42.7 & -4.8 & & & \\
\hline 4 & W18 & 3 & & oct & 1772 & $\mathrm{X}$ & 43.2 & -1.3 & approximate & 244 & \\
\hline 4 & U2 1 & 1 & & & 585 & $\mathrm{x}$ & 42.7 & -0.3 & uncertain & 13 & \\
\hline 4 & $\mathrm{U} 21$ & 3 & & Jul & 678 & $X$ & 42.7 & +0.3 & uncertain & 156 & \\
\hline 4 & T22 & 3 & 2 & Feb & 1374 & $\mathrm{X}$ & 42.4 & +0.8 & uncertain & 54 & \\
\hline 10 & T'25 & 3 & 15 & May & 1427 & $\mathrm{X}$ & 42.2 & +2.5 & approximate & 74 & \\
\hline 10 & $\mathrm{~T} 25$ & 3 & 2 & Feb & 1428 & $\mathrm{X}$ & 42.2 & +2.5 & approximate & 75 & \\
\hline 10 & $\mathrm{R} 25$ & 5 & 22 & Oct & 1912 & $\mathrm{X}$ & 41.5 & 2.3 & approximate & 1166 & \\
\hline 11 & K 02 & 3 & 26 & Jan & 1531 & XI & 38.7 & -9.2 & approximate & 113 & \\
\hline 13 & E04 & 2 & & & 881 & XI & 37.5 & -8.0 & meertain & 15 & \\
\hline 13 & E05 & 3 & 27 & Dec & 1722 & $\mathrm{XI}$ & 37.1 & -7.7 & approximate & 170 & \\
\hline 13 & $\mathrm{H} 03$ & 5 & 23 & Apr & 1909 & $\mathrm{X}$ & 37.8 & -8.9 & acceptable & 990 & \\
\hline 14 & E 09 & 3 & 5 & Apt & 1504 & $\mathrm{XI}$ & 37.4 & -5.6 & approximate & 86 & \\
\hline 15 & $\mathrm{D} 12$ & 3 & & Jan & 1494 & $\mathrm{x}$ & 36.7 & -4.4 & approximate & 85 & \\
\hline 15 & D 13 & 4 & 25 & Dec & 1884 & $\mathrm{X}$ & 37.0 & -3.8 & acceptable & 670 & \\
\hline 15 & 1013 & 6 & 29 & Mar & 1954 & $(\mathrm{~m}=-7.5)$ & 37.0 & -3.6 & acceptable & 3052 & $h_{l}==652$ \\
\hline 15 & $\mathrm{D} 15$ & 3 & 19 & Apr & 1550 & $\mathrm{X}$ & 36.8 & -2.5 & approximate & 116 & \\
\hline 16 & K11 & 3 & 18 & Dec & 1395 & $\mathrm{XI}$ & 39.0 & -0.6 & uncertain & 60 & \\
\hline 17 & $\mathrm{M} 29$ & 3 & 20 & Oet & 1654 & $\mathrm{X}$ & 40.0 & +4.1 & approximate & 146 & \\
\hline 18 & В02 & 3 & 1 & Nov & 1755 & $\mathrm{XI}$ & 36.0 & --9.0 & uncertain & 190 & \\
\hline 19 & A 09 & 5 & 21 & Jan & 1909 & $\mathrm{X}$ & 35.5 & -5.6 & approximate & 969 & \\
\hline 21 & B19 & 3 & 23 & Oct & 1790 & $\mathrm{X}$ & 35.7 & -0.6 & approximate & 278 & \\
\hline 22 & $\mathrm{C} 23$ & 6 & 9 & Sep & 1954 & $\mathrm{X}$ & 36.1 & -1.5 & acceptable & 3068 & $\mathrm{~m}=6.8$ \\
\hline 22 & $\mathrm{C} 24$ & 4 & 15 & Jan & 1891 & $\mathrm{X}$ & 36.5 & +1.8 & approximate & 721 & \\
\hline 22 & B27 & 5 & 24 & Jun & 1910 & $\mathrm{XI}$ & 36.0 & +3.2 & acceptahle & 1055 & \\
\hline
\end{tabular}


of Table V. In brackets are written the estimated values for several intermediate cases for which no information is available. The probable frequency for 50 years, from the applied formula, or percentage, is not adequate for declucing directly the return period, because these periods should be affected by weithing and thus they would be lower than those shown in above mentioned Table $V$.

Table V - Probable Return period for Every REgion.

\begin{tabular}{|c|c|c|c|c|c|c|}
\hline Region & $\begin{array}{c}\text { VII } \\
(60)\end{array}$ & $\begin{array}{c}\text { VIII } \\
(160)\end{array}$ & $\underset{(660)}{\operatorname{Ix}}$ & $\begin{array}{c}x \\
(1360)\end{array}$ & $\begin{array}{c}\text { XI } \\
(2360)\end{array}$ & Remarks \\
\hline 1 & 30 & 一 & - & - & 一 & \\
\hline 2 & 60 & - & - & - & - & \\
\hline 3 & 60 & 160 & - & - & - & \\
\hline 4 & 8 & 23 & (133) & 453 & 2360 & (133) estimate \\
\hline 5 & 60 & - & $\ldots$ & - & 一 & \\
\hline 6 & 30 & 160 & - & - & $\cdots$ & \\
\hline 7 & 60 & 160 & - & - & - & \\
\hline 8 & - & - & 1360 & - & 一 & uncertain \\
\hline 9 & 30 & - & - & - & - & \\
\hline 10 & 30 & 80 & 220 & 680 & - & \\
\hline 11 & 15 & 53 & 660 & $(1366)$ & 2360 & (1366) estimate \\
\hline 12 & $(33)$ & 160 & - & - & - & (33) estimate \\
\hline 13 & (18) & $(91)$ & $(453)$ & 1360 & 1180 & ( ) estimate \\
\hline 14 & 60 & $(216)$ & 660 & - & - & (216) estimate \\
\hline 15 & 7 & 15 & 330 & 453 & - & \\
\hline 16 & 5 & 33 & 330 & $(872)$ & 2360 & (872) estimate \\
\hline 17 & - & 一 & - & 1360 & 一 & uncertain \\
\hline 18 & 60 & 160 & 330 & -- & 一 & \\
\hline 19 & 30 & 160 & $(627)$ & 1360 & - & (627) estimate \\
\hline 20 & 10 & 160 & 660 & $\ldots$ & $\cdots$ & \\
\hline 21 & 30 & $(108)$ & 330 & 1360 & $\cdots$ & (108) estimate \\
\hline 22 & 5 & 9 & 220 & 680 & 一 & \\
\hline area & 0.9 & 3 & 39 & 91 & 337 & \\
\hline
\end{tabular}


In order to check these results by means of the actual intervals, we have computed the return periods from the first up to last earthquakes included in the information, for each intensity regree:

\begin{tabular}{|c|c|c|c|c|c|}
\hline \multirow{2}{*}{$\mathrm{I}_{0}$} & \multicolumn{2}{|c|}{ ordinal } & \multirow{2}{*}{$\begin{array}{c}\text { interval } \\
\text { years }\end{array}$} & \multirow{2}{*}{$\begin{array}{c}\text { occurred } \\
\text { sliocks }\end{array}$} & \multirow{2}{*}{$\begin{array}{l}\text { return } \\
\text { period }\end{array}$} \\
\hline & first & last & & & \\
\hline $\mathrm{XI}^{\circ}$ & 881 & 1910 & 1029 & 7 & 145 \\
\hline $\mathrm{X}^{\circ}$ & 949 & 1954 & 1005 & 16 & 63 \\
\hline IX' & $1 \pm 20$ & 1887 & 467 & 17 & 28 \\
\hline VIIIo & 1804 & 1960 & 164 & 53 & 3 \\
\hline $\mathrm{VII}^{\circ}$ & 1908 & 1960 & 52 & 68 & 0.8 \\
\hline
\end{tabular}

The comparison of these results with those of Trable $\mathrm{V}$ gives a complet conformity for VII and VII' small differences for IXo and for the highest degrees, values separated, approximately, a 30 pereent for Xo and 57 pereent for XI dg, respectively. We think, this is a confirmation of the assumption discussed in EF about a restrictive criterion for classifying the great earthquakes corresponding to old epochs; perhaps they were classified between one and two degrees too low. Thus, we believe it is reasonable to accept the following as probable and approximate values for the return period of the destructive earthquakes, for the whole investigated area,

$\begin{array}{lccccccl}\text { intensity } & = & \text { VII } & \text { VIII } & \text { IX } & \text { X } & \text { XI } & \text { (degrees) } \\ \text { return } & = & 1 & 3 & 30 & 80 & 240 & \text { (years) }\end{array}$

This list suggests, only as an approximate probability, a violent earthquake to be espected in the area during a half of a century.

Final no'ite.

This paper is continuation of the seismic studies on the Peninsula Iberiea area and will be followed by other treating the liberated energy and seismic flux. This report has been subsided by the Air Foree Office of Scientific Research, OAR, through the European Office of Aerospace Research of United States Lir Forces, contract $A F$ 61 (052)-657, as part of the Adraneed Researeh Project Ageney's Project VELA-UNIFORM. 


\section{REFERENCES}

(1) V.V.Beroussov, Carle seismologique de l'Europe Occidental, Moseow, 1963.

(2) France, Recommandations is, 1955.

$\left({ }^{3}\right)$ Greece, Gode of earlhquakes resisting building worlis, 1959.

(') Japan, slandards of aseismic eivil engineering construetion, 1952.

(5) J. M. Munuera, Estudio previo para el calculo de construeciones sismo. resistenles, I.G.C., Madrid, 1962.

(i) .. M. Munuera, Seismic Dala, Mem. I.G.C., Madrid, 1963.

(7) J. M. Munuena, Epicenters and frequency. Mem. I.G.C., Madrid, 1964.

${ }^{(8)}$ Portugal, Portuguese code on earthqualie resisting struchure, 1958.

$\left.{ }^{9}\right)$ J. P. Rotris, Le risque seismique et la recherche d'une protection contre les seismes, "Nature ", 3341-42.

(10) R. SoETAd, Seismic zones in Indonesia, Djaliarta, 1962.

(11) J. М. IU Unera, Un mapa espanol de freouencia sismica probable, UNESCO meeting of Seismology and earthquake engineering, Paris, Apr. 1964. 